\title{
Acute exposure to silica nanoparticles aggravate airway inflammation: different effects according to surface characteristics
}

\author{
Hye Jung Park ${ }^{1}$, Jung-Ho Sohn ${ }^{1,2}$, Yoon-Ju Kim ${ }^{1}$, Yoon Hee Park ${ }^{1}$, Heejae Han ${ }^{1}$, Kyung Hee Park ${ }^{1}$, \\ Kangtaek Lee ${ }^{3}$, Hoon Choi ${ }^{3}$, Kiju Um ${ }^{3}$, In-Hong Choi ${ }^{4}$, Jung-Won Park ${ }^{1}$ and Jae-Hyun Lee ${ }^{1}$
}

Silica nanoparticles (SNPs) are widely used in many scientific and industrial fields despite the lack of proper evaluation of their potential toxicity. This study examined the effects of acute exposure to SNPs, either alone or in conjunction with ovalbumin (OVA), by studying the respiratory systems in exposed mouse models. Three types of SNPs were used: spherical SNPs (S-SNPs), mesoporous SNPs (M-SNPs), and PEGylated SNPs (P-SNPs). In the acute SNP exposure model performed, 6-week-old BALB/c female mice were intranasally inoculated with SNPs for 3 consecutive days. In the OVA/SNPs asthma model, the mice were sensitized two times via the peritoneal route with OVA. Additionally, the mice endured OVA with or without SNP challenges intranasally. Acute SNP exposure induced significant airway inflammation and airway hyper-responsiveness, particularly in the S-SNP group. In OVA/SNPs asthma models, OVA with SNP-treated group showed significant airway inflammation, more than those treated with only OVA and without SNPs. In these models, the P-SNP group induced lower levels of inflammation on airways than both the S-SNP or M-SNP groups. Interleukin (IL)-5, IL-13, IL-1 $\beta$ and interferon- $\gamma$ levels correlated with airway inflammation in the tested models, without statistical significance. In the mouse models studied, increased airway inflammation was associated with acute SNPs exposure, whether exposed solely to SNPs or SNPs in conjunction with OVA. P-SNPs appear to be relatively safer for clinical use than S-SNPs and M-SNPs, as determined by lower observed toxicity and airway system inflammation.

Experimental \& Molecular Medicine (2015) 47, e173; doi:10.1038/emm.2015.50; published online 17 July 2015

\section{INTRODUCTION}

Nanotechnology is a recently developing field that allows for the production of nanoparticles (NPs) at supramolecular levels, typically $<100 \mathrm{~nm}$. Nanotechnology has been applied to many industrial fields, including textiles, cosmetics, food packaging and medicines. In particular, the application of nanotechnology in the medical field has improved various biotechnological processes such as drug synthesis and their targeted delivery. Nanotechnology is expected to progress and expand in the future. ${ }^{1-4}$ The increasing applications of nanotechnology warrant an increased study of the toxicity of NPs, with emphasis on their potential safety issues. Studies thus far have revealed that NPs can alter immune function and cause inflammation in various organs and systems. The toxicity of NPs, particularly associated with the liver, kidney, lung and immune system, has been clearly established. ${ }^{5-10}$ These toxicity studies and their results have been helpful for determining the concentration, size and surface pattern of NPs that should be selected for safe application.

Of the various clinical routes of NP administration, inhalation is the main route of exposure due to the small size of NPs and their ease of airborne dispersion. Humans can be easily exposed to NPs unintentionally via aerosolization. Recent clinical studies have shown that occupational exposure during the manufacturing of NPs can cause respiratory diseases such as asthma and bronchitis. ${ }^{11-13}$ Therefore, airway toxicity related to NPs has garnered considerable recent attention. Asthma, a chronic inflammatory airway disease characterized by variable bronchial obstruction, is a common, highly prevalent and significant airway disease. There are

\footnotetext{
${ }^{1}$ Division of Allergy and Immunology, Department of Internal Medicine, Yonsei University College of Medicine, Severance Hospital, Seoul, Korea; ${ }^{2}$ Department of Life Science, Research Institute for Natural Sciences, Hanyang Biomedical Research Institute, Hanyang University, Seoul, Korea; ${ }^{3}$ Department of Chemical and Biomolecular Engineering, Yonsei University, Seoul, Korea and ${ }^{4}$ Department of Microbiology, Brain Korea 21 Project for Medical Science, Institute for Immunology and Immunological Diseases, Yonsei University College of Medicine, Seoul, Korea

Correspondence: Professor J-H Lee, Division of Allergy and Immunology, Department of Internal Medicine, Yonsei University College of Medicine, Severance Hospital, 50 Yonsei-ro, Seodaemun-gu, Seoul 120-752, Korea. 
many risk factors for developing or aggravating asthma and asthma-like airway diseases such as air pollutants, cigarette smoking and viral infection. ${ }^{14-16}$ These factors, as well as fine materials like NPs, may induce and aggravate asthma. Many studies seeking to understand and reduce the toxicity associated with NPs, have sought to examine them in more detail, as well as their characteristics, such as surface charge and surface pattern, which contribute to airway inflammation. ${ }^{17-19}$

Silica, or silicon oxide $\left(\mathrm{SiO}_{2}\right)$, is naturally found as sand or quartz. Silica is a main component of glass and concrete. Owing to its optical, thermodynamic characteristics and chemical durability, silica has been used as a heat-resistant glass and semiconductor; it is commonly used in optical communications systems and ceramic technology. However, silica is a well-known cause of pneumoconiosis, often presenting as chronic bronchitis, emphysema and lung fibrosis. ${ }^{20,21}$ In addition, lung cancer has been associated with silicosis, which is induced by inhalation of silica. ${ }^{22}$ Silicosis may cause various autoimmune disorders, including rheumatoid arthritis, systemic lupus erythematosus, systemic sclerosis and vasculitis. $^{23,24}$ Due to these potential health risks, the use of silica should be carefully regulated and handled with proper safety precautions.

Silica NPs (SNPs) have recently been used in biosciences, medicines and daily life, after studies have revealed that SNPs are relatively safe. ${ }^{25-28}$ Unfortunately, some studies have since revealed inhalant-associated toxicity of SNPs in chronic exposure conditions; ${ }^{29,30}$ however, the acute effects of SNPs on airway systems has not yet been fully evaluated in vivo. Especially, aggravating effects of SNPs on airway systems in asthma mice model induced by ovalbumin (OVA) sensitization and challenges has not been well known. Moreover, there are few studies examining a counterplan for reducing toxicity related to SNPs. In this study, exposure to SNPs alone as well as in conjunction with OVA was induced to determine the toxicity of these NPs on airway systems and examine any associated aggravation of airway inflammation. Furthermore, various surface patterns were studied to determine a possible, relatively safe SNP with reduced toxicity.

\section{MATERIALS AND METHODS}

\section{Preparation and characterization of SNPs}

This study examined three types of SNPs: spherical (S-SNPs), mesoporous (M-SNPs) and PEGylated (P-SNPs; PEG, polyethylene glycol). To prepare the S-SNPs, $11.87 \mathrm{ml}$ of ethanol (Sigma-Aldrich, St. Louis, MO, USA), $2.1 \mathrm{ml}$ of distilled (DI) water, $0.67 \mathrm{ml}$ of tetraethoxysilane (Sigma-Aldrich) and $0.36 \mathrm{ml}$ of $5 \mathrm{M} \mathrm{NH} \mathrm{NH}_{4} \mathrm{OH}$ (Sigma-Aldrich) were mixed in a vial and stirred for $24 \mathrm{~h}$.

M-SNPs were synthesized using $53.4 \mathrm{~g}$ of DI water, $6.24 \mathrm{~g}$ of cetyltrimethylammonium chloride (Sigma-Aldrich) and $0.3 \mathrm{~g}$ of sodium acetate (Sigma-Aldrich), were mixed well, and then heated in a $65^{\circ} \mathrm{C}$ oil bath. After heating for $1 \mathrm{~h}, 3.9 \mathrm{ml}$ of tetraethoxysilane was slowly added to the mixture and then stirred for $24 \mathrm{~h}$. The resulting suspensions were purified by centrifugation, re-dispersed in ethanol and DI water, and then finally re-dispersed in DI water.

P-SNPs were prepared by surface functionalization of S-SNP. Briefly, thiol-functionalized S-SNP was prepared by adding (3-mercaptopropyl) trimethoxysilane (10\% of tetraethoxysilane) $5 \mathrm{~h}$ after the addition of $\mathrm{NH}_{4} \mathrm{OH}$ during synthesis of S-SNP. After purification, these particles were conjugated with maleimidefunctionalized PEG (molecular weight: 2000) in DI water for $24 \mathrm{~h}$ (weight ratio: $\sim 0.5: 1$ ). Excess amount of PEG was removed by centrifugation and dispersed in DI water to obtain P-SNP.

\section{Visualization and surface area calculation of SNPs}

To characterize the SNPs, transmission electron microscopy was performed using a Tecnai 20 (FEI Co., Hillsboro, OR, USA). The SNPs were diluted to a concentration of $1 \mathrm{mg} \mathrm{ml}^{-1}$ in a phosphatebuffered saline solution. Transmission electron microscopy specimens of the diluted SNPs were prepared by placing one drop on a carboncoated copper grid, which was allowed to dry at $60^{\circ} \mathrm{C}$ for $15 \mathrm{~min}$. Transmission electron microscopy analyses were performed at $25 \pm 2^{\circ}$ C; each SNP measured $\sim 100 \mathrm{~nm}$ (Figure 1). These analyses indicated that S-SNPs and M-SNPs had spherical and mesoporous morphologies, respectively. The P-SNPs also demonstrated spherical morphology.

Nitrogen sorption experiments were performed at $77 \mathrm{~K}$ with the Micromeritics ASAP 2020 system (Micromeritics, Norcross, GA, USA). Specific surface area and pore size of the particles were calculated using the Brunauer-Emmett-Teller equation and the Barrett-Joyner-Halenda method, based on the desorption branch, respectively.

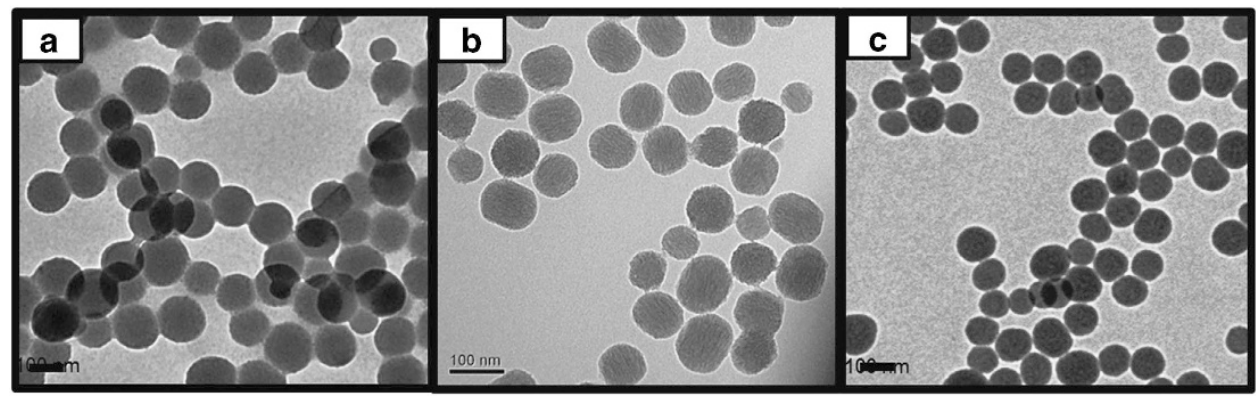

Figure 1 Transmission electron microscopy images of (a) S-SNPs, (b) M-SNPs and (c) P-SNPs. M-SNP, mesoporous silica nanoparticle; P-SNP, PEGylated silica nanoparticle; S-SNP, spherical silica nanoparticle. 
Animals

Female BALB/c mice, between 5 and 6 weeks old, were used in this study (Orient, Daegeon, Korea). All mice were maintained at conventional animal facilities under specific, pathogen-free conditions (room temperature of $21-24{ }^{\circ} \mathrm{C}$, relative humidity between $45 \%$ and $70 \%$, with a 12-h light/dark cycle). All experimental protocols were approved by the Animal Research Ethics Board of Yonsei University (Seoul, Korea).

\section{Direct acute exposure to SNPs}

To establish the effects of SNPs in the airway system, the mice were intranasally inoculated with SNPs (S-SNPs, M-SNPs and P-SNPs in S-SNP group, M-SNP group and P-SNP group, respectively) or saline on days 0,1 , and 2 (Figure $2 \mathrm{a}$ ). All mice were killed $48 \mathrm{~h}$ after the last intranasal inoculation.

\section{Establishment of OVA-induced acute asthma model with SNP exposure (OVA/SNPs asthma model)}

To make acute asthma mice model, mice were sensitized with an intraperitoneal injection of either $200 \mu \mathrm{l}$ of $10 \mu \mathrm{g}$ OVA (SigmaAldrich) suspended in 1\% aluminum hydroxide (Resorptar, Indergen, NY, USA) or $200 \mu \mathrm{l}$ of saline on days 1 and 14. On days 21, 22 and 23, the OVA-sensitized mice were challenged intranasally with $30 \mu \mathrm{l}$ of OVA $\left(1 \mathrm{mg} \mathrm{ml}^{-1}\right)$ in saline solution. SNPs (S-SNPs, M-SNPs and $\mathrm{P}-\mathrm{SNPs}$ in S-SNP group, M-SNP group and P-SNP group, respectively) or saline were co-administered by intranasal inoculation of $200 \mu \mathrm{g}$ (Figure 2b). All mice were killed $48 \mathrm{~h}$ after the last intranasal inoculation.

\section{Measurement of airway hyper-responsiveness}

Airway hyper-responsiveness (AHR) in response to inhaled aerosolized methacholine was measured using a forced oscillation technique (FlexiVent; SCIREQ, Montreal, QC, Canada) $48 \mathrm{~h}$ after the last intranasal inoculation. Cannulas were inserted into anesthetized mice via tracheostomy, after which the mice were connected to a ventilator. Aerosolized phosphate-buffered saline or methacholine at varying concentrations, $3.125,6.25,12.5,25.0$ and $50.0 \mathrm{mg} \mathrm{ml}^{-1}$, was diffused to the mice for $10 \mathrm{~s}$ via a nebulizer. AHR was measured and continuously recorded for up to $2 \mathrm{~min}$.

\section{Bronchoalveolar lavage and inflammatory cell counting}

To collect bronchoalveolar lavage fluid (BALF), lung lavage was performed three times by passing $1 \mathrm{ml}$ of Hank's Balanced Salt Solution through the tracheal tube. The recovered BALF was
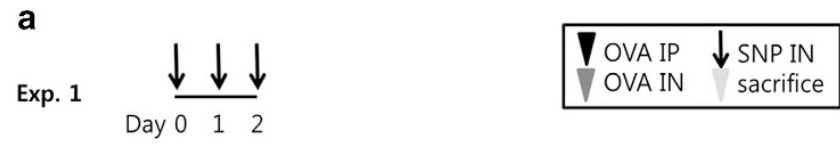

b

Exp. 2

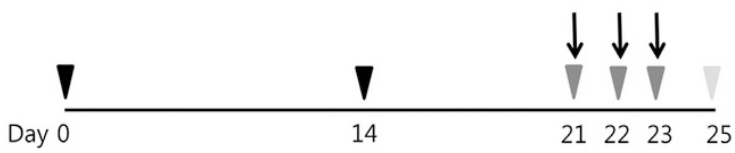

Figure 2 Experiment scheme. (a) Experiment 1: acute SNP exposure models. (b) Experiment 2: acute exposure to SNPs with OVA-induced asthma models. exp, experiment; IN, intranasal inoculation; IP, intraperitoneal injection; OVA, ovalbumin; SNP, silica nanoparticle. centrifuged at $4{ }^{\circ} \mathrm{C}$ for $3 \mathrm{~min}$ at $1500 \mathrm{~g}$. The cellular pellet was resuspended in $300 \mu \mathrm{l}$ Hank's Balanced Salt Solution. One part of the resuspended BALF cells $(250 \mu \mathrm{l})$ were centrifuged by cytocentrifugation (Cytospin 3; Thermo, Waltham, MA, USA) at $4{ }^{\circ} \mathrm{C}$ for $5 \mathrm{~min}$ at 1000 r.p.m. and were pelleted to cytospin slides. The slides were stained with hematoxylin and eosin (Hemacolor, Merck, Darmstadt, Germany) and counted the differential inflammatory cells including neutrophils, eosinophils, lymphocytes and macrophages (200 cells per cytospin). The remaining resuspended BALF cells $(50 \mu \mathrm{l})$ were used for counting the total number of cells using a hemocytometer and trypan blue staining.

\section{Measurement of cytokine levels}

Concentrations of interleukin (IL)-5, IL-13, IL-1 $\beta$ and interferon- $\gamma$ in the lung tissues were assessed by ELISA (R\&D Systems, San Diego, CA, USA) according to the manufacturer's instructions. The ELISA detection ranges for IL-5, IL-13, IL-1 $\beta$ and interferon- $\gamma$ are 31.3-2000, $62.5-4000,15.6-1000$ and $31.3-2000 \mathrm{pg} \mathrm{ml}^{-1}$, respectively.

\section{Histological analysis}

After the use of one lung in the collection of BALF, the other lung was fixed in $4 \%$ formalin and embedded in paraffin. Lung sections were cut into 3- to 4- $\mu \mathrm{m}$ thick segments and stained with hematoxylin and eosin and periodic acid-Schiff for histological analysis. The slides were observed under light microscope.

\section{Statistical analysis}

All results are expressed as the mean \pm s.e.. One-way analysis of variance was performed using SPSS statistical software version 12.0 (SPSS Inc., Chicago, IL, USA). The AHR data were analyzed with repeated analysis of variance measures, followed by a post hoc Bonferroni test. The remaining data were analyzed with one-way analysis of variance followed by a post hoc Bonferroni test. $P$-values $<0.05$ were considered statistically significant.

\section{RESULTS}

Difference and chemical characteristics of three kinds of SNPs

The surface area calculated using the Brunauer-Emmett-Teller equation of S-SNPs and M-SNPs was 12.7 and $70.6 \mathrm{~m}^{2} \mathrm{~g}^{-1}$, respectively. The surface area of P-SNPs was similar to those of S-SNPs. The average pore diameter of the M-SNPs was calculated to be $3.0 \mathrm{~nm}$ using the Barrett-Joyner-Halenda method.

\section{Effects of acute SNP exposure}

Significant airway inflammation was observed for each type of SNP treatment; however, the level of inflammation varied according to the surface characteristics of the SNP used. The results of BALF examination revealed statistically significant variation in the differential cell counts performed on the S-SNP group as compared with the control group, including the total cell count, macrophage count and neutrophil count. Lymphocytes were significantly higher in the S-SNP group than in the other groups. The M-SNPs and P-SNP groups also demonstrated higher total cell and macrophage counts than did the control group, but without statistical significance. The M-SNP 
and P-SNP groups induced a smaller increase in cell count than did the S-SNP group (Figure 3a).

Significantly higher AHR was observed in the S-SNP group than in the control group. The M-SNP and P-SNP groups also demonstrated higher AHR than did the control group, but lower than the AHR observed in the S-SNP group. No statistically significant differences were observed (Figure $3 b$ ).

In each of the SNP groups, histologic examination revealed higher peribronchial and perivascular inflammation than that observed in the control group. The S-SNP group demonstrated marked increase in bronchial epithelial thickness with abundant inflammatory cell infiltration. The histologic findings between M-SNP and P-SNP groups could not be visually discriminated (Figure 4).

Higher levels were obtained for the S-SNP group than the control group for each cytokine measured, including IL-5, IL-13, IL- $1 \beta$ and INF- $\gamma$. The levels of IL- 5 and IL-13, which are known as helper T cell type 2 (Th2) cytokines, were lower in the P-SNP group than in the S-SNP group; however, none of the cytokine measurements demonstrated a statistically significant difference (Figure 5).
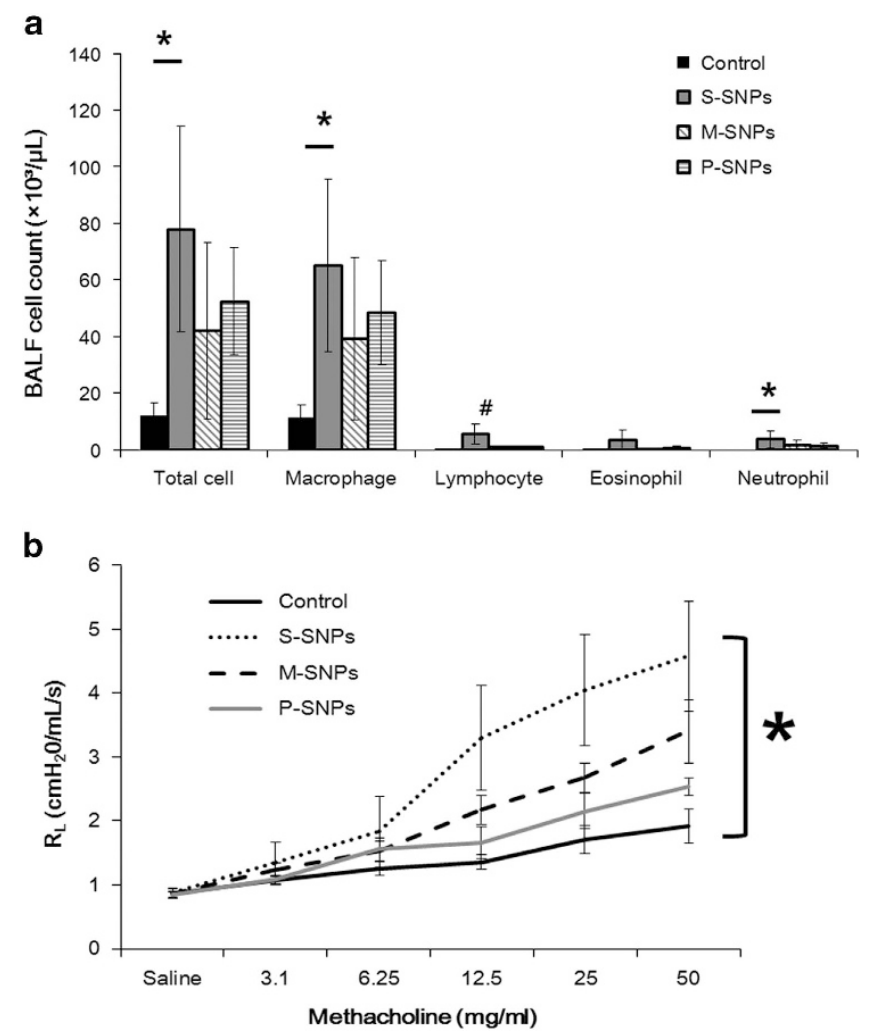

Figure $\mathbf{3}$ The airway inflammation as demonstrated in (a) bronchoalveolar lavage fluid (BALF) and (b) airway hyperresponsiveness in acute SNP exposure models. ${ }^{*} P<0.05$ between two variables; ${ }^{\#} P<0.05$ compared with others. M-SNP, mesoporous silica nanoparticle; P-SNP, PEGylated silica nanoparticle; $\mathrm{R}_{\mathrm{L}}$, resistance of lung; S-SNP, spherical silica nanoparticle.
Effects of acute SNP exposure in the OVA-induced asthma model

SNPs, inoculated thrice in conjunction with OVA, induced asthma in the mice. Upon examination of the BALF, the total cell counts in the S-SNP and M-SNP groups were significantly higher than that in the control group. The number of macrophages was significantly higher in the S-SNP and M-SNP groups than in all of the other groups-the control group, OVA-only asthma group and P-SNP group. Eosinophils were significantly elevated in the S-SNP and M-SNP groups than in the control group. The total number of cells and eosinophils were higher in the P-SNP group than in the control group, but lower compared with those of the S-SNP and M-SNP groups (Figure 6a).

AHR measurements in the OVA asthma group were greater than in the control group, yet without statistically significant meaning. Overall, no significant difference in AHR was observed among the various SNP groups (Figure 6b).

Significantly more cellular infiltration in peribronchial and perivascular tissues was observed in the OVA asthma group, as well as in the three SNP groups, as compared with the control group. No statistical difference was observed among the OVA asthma group and each of the SNP groups (Figure 7).

IL-5 and IL-13 levels were statistically significantly greater in the OVA asthma group and SNP groups than in the control group. No significant statistical difference was noted between the OVA asthma group and each of the SNP groups. Similarly, the levels of IL- $1 \beta$ and INF- $\gamma$ were higher in the OVA asthma group and SNP groups than in the control group; however, no statistically significant difference was observed. The P-SNP group demonstrated lower levels of IL-5, IL-13, IL-1 $\beta$ and INF- $\gamma$ than the OVA asthma and other SNP groups, except the control group (Figure 8).

\section{DISCUSSION}

This study demonstrated that acute exposure, simulated just once per day for 3 days, can harm the respiratory system. Acute SNP exposure, as demonstrated here, can induce significant airway inflammation and aggravate allergic airway inflammation in the OVA-induced asthma models.

In this study, short-term exposure to SNPs induced respiratory inflammation with associated AHR in each of the S-SNP, M-SNP and P-SNP groups. Both BALF and histologic examination showed significantly higher cell counts in the S-SNP group than in the control group. Acute exposure to SNPs in conjunction with OVA (OVA/SNPs asthma model) also induced severe airway inflammation when compared with those treated with OVA alone. No significant difference in AHR was observed among all the groups, including mice in the OVA asthma group and OVA/SNPs asthma mice. Both models showed increased levels of Th1 cytokines (IL-1 $\beta$ and interferon- $\gamma$ ) and Th2 cytokines (IL-5, and IL-13), without significant statistical difference. Some studies have shown that Th2 cytokines have a key role in airway toxicity. ${ }^{10,29,30}$ However, in this study, increased macrophage counts in BALF samples and increased levels of IL- $1 \beta$ and interferon- $\gamma$ were 


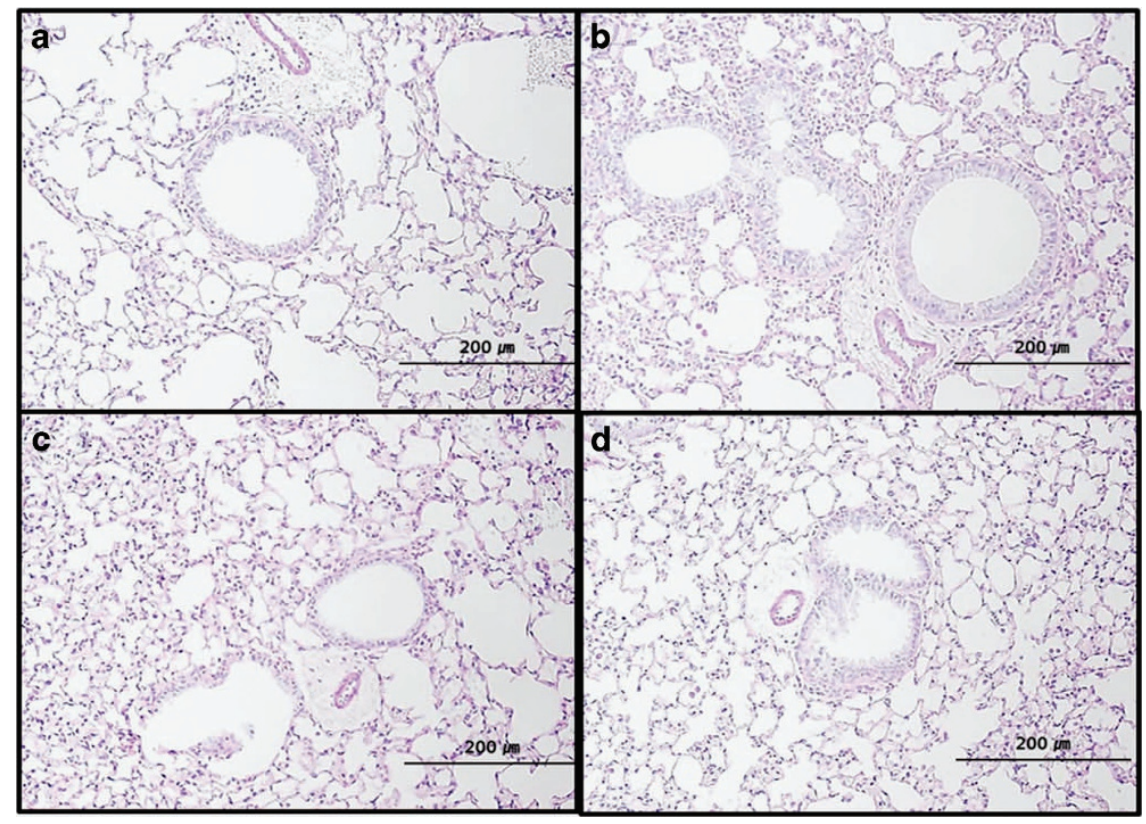

Figure 4 Histological findings of lungs representing peribronchial and perivascular tissues in the (a) control group, (b) S-SNP group, (c) M-SNP group and the (d) P-SNP group. M-SNP, mesoporous silica nanoparticle; P-SNP, PEGylated silica nanoparticle; S-SNP, soluble silica nanoparticle.

a

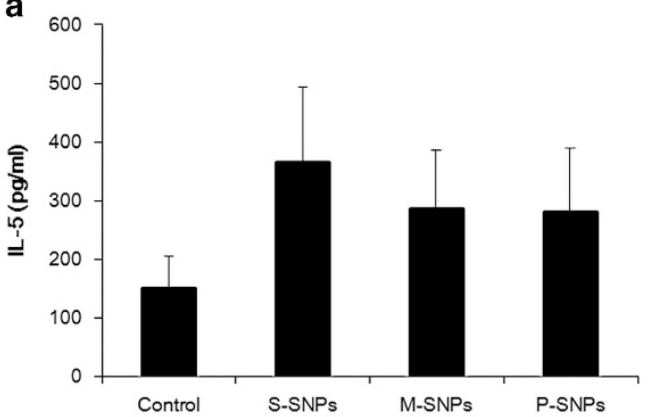

C

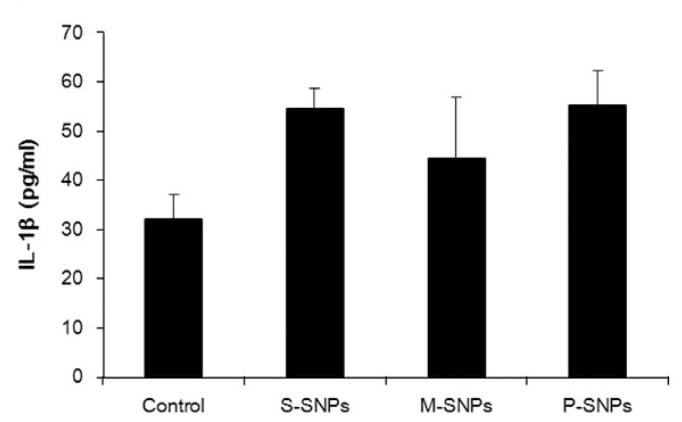

b

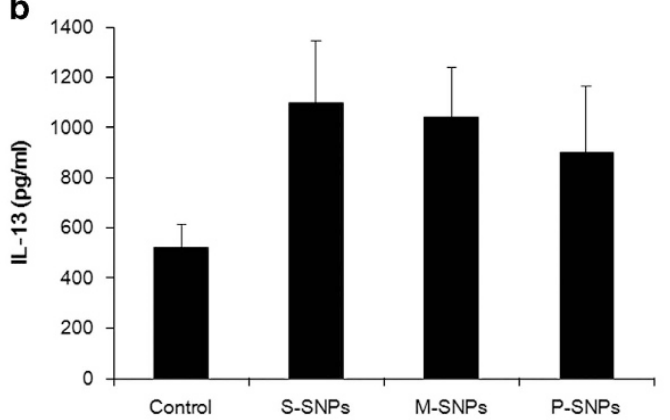

d

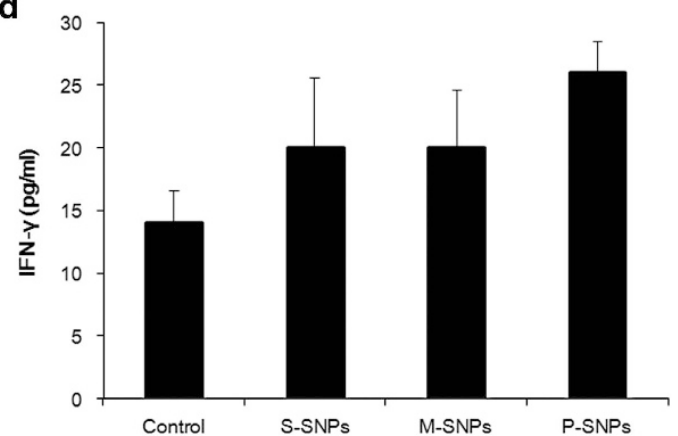

Figure 5 Cytokine levels of (a) IL-5, (b) IL-13, (c) IL-1 $\beta$ and (d) IFN- $\gamma$ in acute SNP exposure models. IFN- $\gamma$, interferon- $\gamma$; IL-5, interleukin5; M-SNP, mesoporous silica nanoparticle; P-SNP, PEGylated silica nanoparticle; S-SNP, spherical silica nanoparticle.

observed, particularly in the SNP models. It could be assumed that this airway inflammation was induced not only via the Th2 pathway but also via the Th1 pathway.

Th1- and Th2-related immunity maintain a delicate balance. If one side increases, the other side naturally decreases. Several
Th1 enhancers reduce Th2-related immunity. ${ }^{31}$ OVA induces Th2-related immunity, whereas SNPs are hypothesized to induce Th1-related immunity. Therefore, OVA/SNP groups will induce a Th2-related response to a lesser extent than that in only OVA-treated groups. Indeed, the AHR and the IL-5 and 

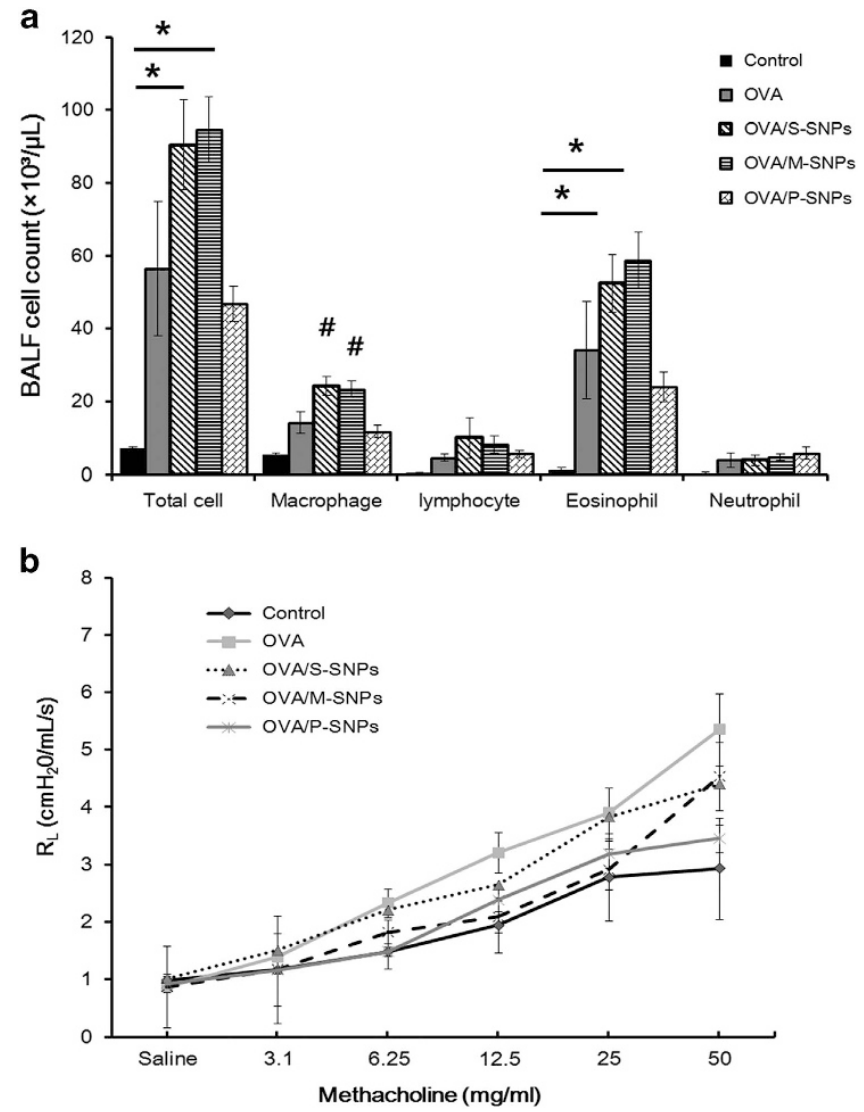

Figure 6 Airway inflammation as determined by analysis of (a) bronchoalveolar lavage fluid (BALF) and (b) airway hyperresponsiveness in OVA/SNPs asthma models. ${ }^{*} P<0.05$ between two variables; ${ }^{\#} P<0.05$ compared with others. M-SNP, mesoporous silica nanoparticle; OVA, ovalbumin; P-SNP, PEGylated silica nanoparticle; $\mathrm{R}_{\mathrm{L}}$, Resistance of lung; $\mathrm{S}-\mathrm{SNP}$, spherical silica nanoparticle.

IL-13 levels in OVA/SNP groups decreased compared with that in the OVA group (Figures $6 \mathrm{~b}$ and 8 ). SNP, a Th1 immunity enhancer, may reduce the Th2-related immunity by lowering AHR and by lowering the levels of IL-5 and IL-13. In contrast, OVA, a Th2 enhancer, may reduce Th1-related immunity. The decrease in macrophage counts in BALF in the OVA/SNP model compared with those in the SNP model is the result of a protective effect of OVA against Th1-related immunity (Figures 3a and 6a). However, overall inflammation based on the total cell count in BALF and pathologic finding revealed that the OVA/SNP model induced severe inflammation. Although not statistically significant, we believe that SNP slightly reduced Th2-related immunity induced by OVA by inducing Th1-related immunity. Therefore, overall total inflammation induced by OVA/SNP is similar to or more severe than that in the OVA- or SNP-treated groups.

We selected a specific dose of SNPs (200 $\mu$ g per mouse) for this study. Previous studies have revealed that there is a dosedependent effect of SNP-induced airway inflammation. ${ }^{19}$ Thus, most current studies use a specific dose of SNP $(12.5-200 \mu \mathrm{g})$ to evaluate its toxicity. ${ }^{32}$ The number of SNP in ambient air range from $2 \times 10^{4}$ to $2 \times 10^{5} \mathrm{~cm}^{-3}$, with mass concentrations of $>50 \mu \mathrm{g} \mathrm{m}^{-3}$ near major highways. ${ }^{13}$ Average breathing volume per day is approximately $15-25 \mathrm{~m}^{3}$. An individual working near a major highway might therefore inhale anywhere between 750 and $1250 \mu \mathrm{g}$ of SNP in a day. In mouse experiments of toxicity to a specific material, 10- to 1000-fold of what is considered a toxic dose of the study compound to be invesigated is typically used. We selected an SNP dose of $\sim 400$-fold, calculated based on the mass of SNP typically inhaled relative to body mass (human: $0.02 \mu \mathrm{gg}^{-1}$; mouse (in this study): $8 \mu \mathrm{g} \mathrm{g}^{-1}$ ). We believe that this SNP dose of $200 \mu \mathrm{g}$ per mouse is clinically feasible. Indeed, these experiments showed significantly increased AHR, which is of clinical importance, inducing clinical signs such as cough and dyspnea under the stimulation of less irritable materials.

Many studies have demonstrated that the toxicity of SNPs depends on several factors, including size, surface area, electric charge and function. ${ }^{33,34}$ Some studies have revealed correlation between the surface pattern of SNPs and the level of toxicity; 2,35 however, results vary according to experimental setting and designs. In this study, the toxicity of acute SNP exposure varied owing to the surface pattern of the SNP. In acute SNP exposure models, the P-SNP and M-SNP groups induced less airway inflammation than the S-SNP group, without statistical significance. Among OVA/SNP asthma mice, those in the P-SNP group induced lesser signs of airway inflammation than those in the S-SNP and M-SNP groups, and the difference was statistically significant. The contact area is a critical factor for inducing toxicity during the interaction between SNPs and tissues. The contact and surface area of M-SNPs is largest among the three kinds of SNPs (M-SNPs, $70.6 \mathrm{~m}^{2} \mathrm{~g}^{-1}$; S-SNPs, $\left.12.7 \mathrm{~m}^{2} \mathrm{~g}^{-1}\right)$. Smaller surface area has lesser opportunity to interact with tissue to induce inflammation. Therefore, the larger surface area of M-SNPs could induce the most severe inflammation compared with the other SNPs in this study. In P-SNPs, the surfaces of S-SNPs are functionalized with the biocompatible PEG ligands. Therefore, P-SNPs exhibited the lowest toxicity among three different types of SNPs.

In summary, the toxicity of SNPs was lower in the P-SNP group than in the S-SNP or M-SNP group. P-SNPs did induce airway inflammation but lesser than did S-SNPs and M-SNPs induce. P-SNPs induce lesser inflammation on the airway, with reduced inflammation in OVA/SNPs asthma models. P-SNPs may be more suitable for use in various medical/pharmaceutical fields, including drug delivery systems, diagnostic carriers and other medical applications. The harmful effects associated with SNPs should be carefully considered before implementation, particularly when exposure of the airway system is possible.

Although various studies have evaluated the toxicity of SNPs, this study cannot be compared with the previous work owing to significant differences in its experimental design. This study focused on examining respiratory system response after shortterm exposure to SNPs, with or without OVA sensitization, as well as the different effects of SNPs according to their surface patterns. These results contribute to the existing knowledge base for SNP usage in scientific and industrial fields. 


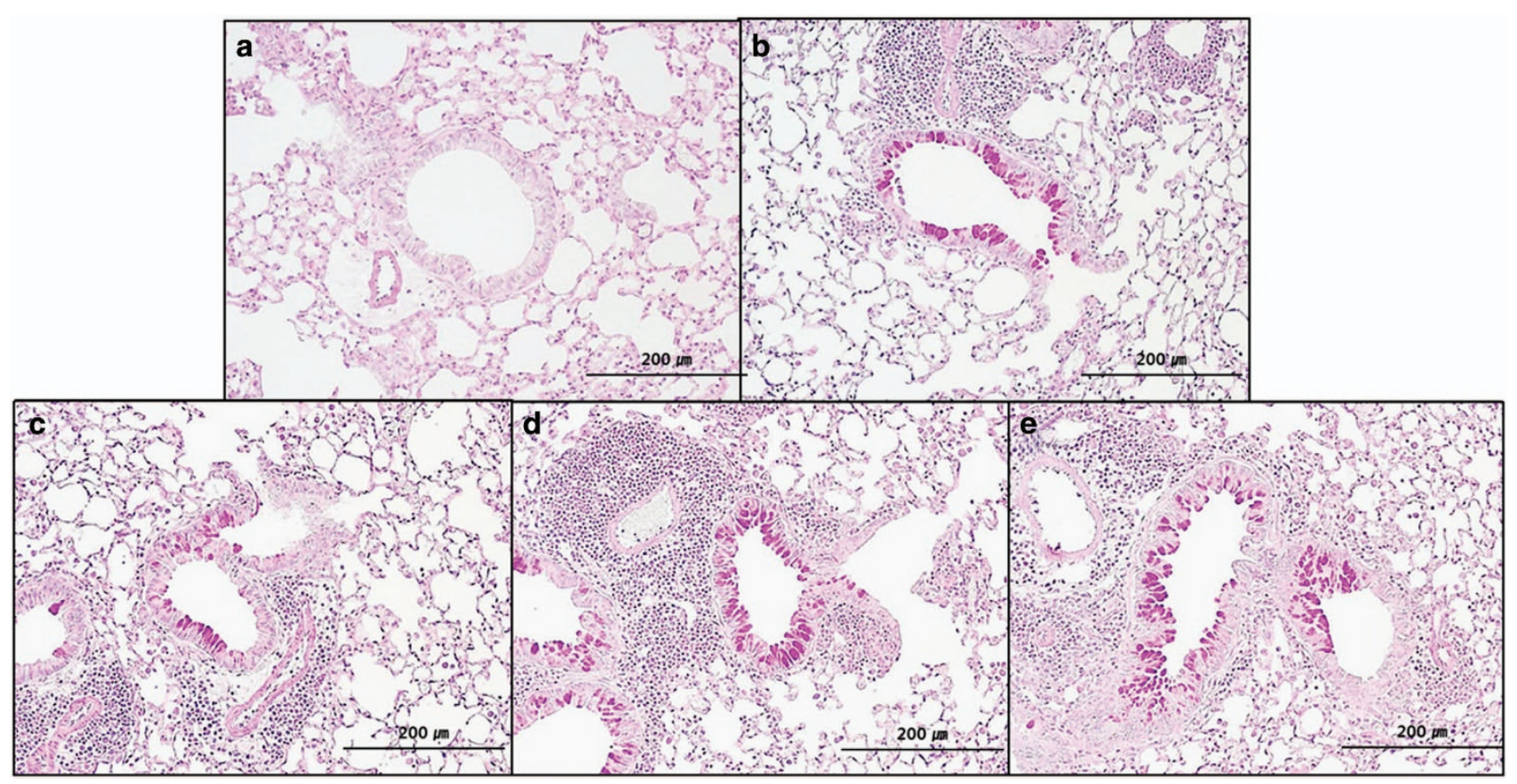

Figure 7 Histological findings in peribronchial and perivascular tissues of the (a) control group, (b) OVA-only group, (c) S-SNP group, (d) M-SNP group and (e) P-SNP group. M-SNP, mesoporous silica nanoparticle; P-SNP, PEGylated silica nanoparticle; S-SNP, spherical silica nanoparticle.
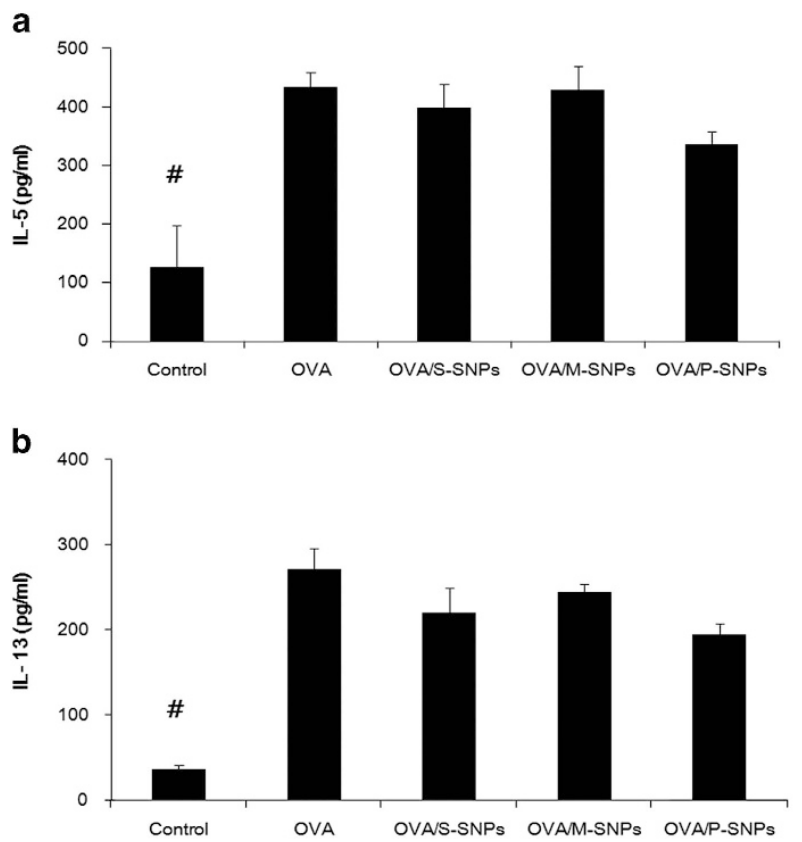

C

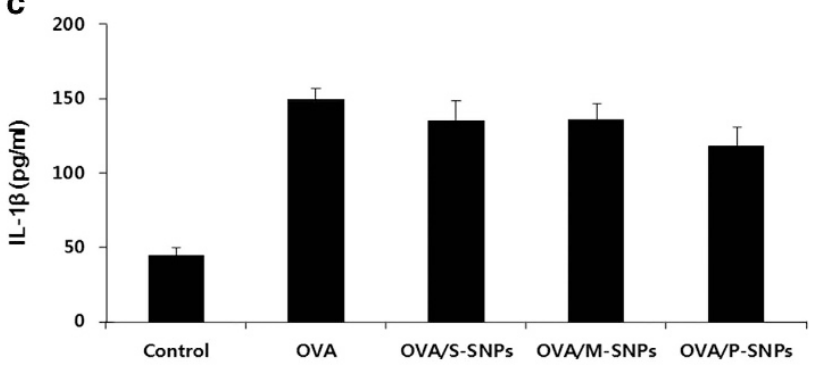

d

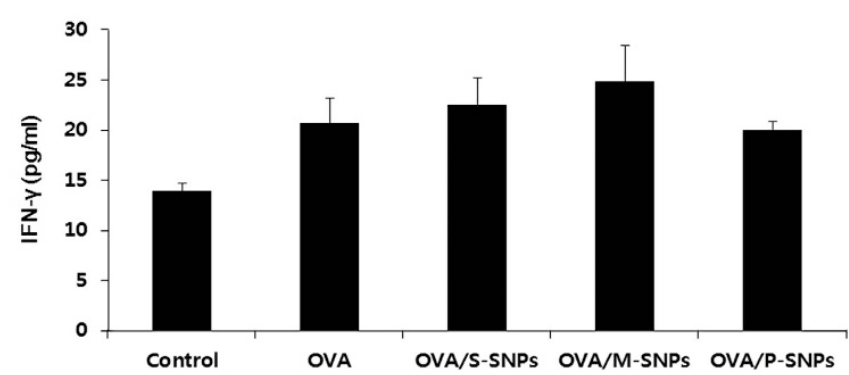

Figure 8 Cytokine levels including (a) IL-5, (b) IL-13, (c) IL-1 $\beta$ and (d) IFN- $\gamma$ in OVA/SNPs asthma models. ${ }^{*} P<0.05$ compared with others. IFN- $\gamma$, interferon- $\gamma$; IL-5, interleukin-5; M-SNP, mesoporous silica nanoparticle; P-SNP, PEGylated silica nanoparticle; S-SNP, spherical silica nanoparticle.

However, the present study did not completely examine the effect of SNPs in conjunction with OVA in the OVA/SNPs asthma models to determine its use as an adjuvant. The OVAonly group demonstrated lower total cell counts and macrophage counts in BALF than the S-SNP and M-SNP groups. As S-SNPs and M-SNPs can induce airway inflammation on their own, it could not be determined whether the increased effects observed in the OVA/SNP asthma models are due to actions of SNPs alone, or because of an adjuvant effect with OVA. To evaluate more accurate pro-inflammatory effects and adjuvant effects of OVA/SNP models, further study is required. Moreover, the present study revealed that Th2 and Th1 cytokines are 
associated with toxic effects of SNPs; however, the mechanisms by which SNPs induce inflammation on the respiratory system have not yet been fully evaluated. A more accurate evaluation of this mechanism, as well as further cytokine and gene studies, is required.

In conclusion, this study demonstrated the toxic effects of acute SNP exposure. Acute SNP exposure can induce significant airway inflammation, and further aggravate airway inflammation, as demonstrated in the OVA asthma model. In terms of surface characteristics, P-SNPs appear to be safer for clinical use than S-SNPs or M-SNPs.

\section{CONFLICT OF INTEREST}

The authors declare no conflict of interest.

\section{ACKNOWLEDGEMENTS}

This study was supported by a faculty research grant of Yonsei University College of Medicine for 2013(6-2013-0024) and Nano-Material Technology Development Program through the National Research Foundation of Korea (NRF) funded by the Ministry of Education, Science and Technology (No. 2009-0082417).

1 Roco MC. Nanotechnology: convergence with modern biology and medicine. Curr Opin Biotechnol 2003; 14: 337-346.

2 Vallet-Regi M, Balas F, Arcos D. Mesoporous materials for drug delivery. Angew Chem Int Ed Engl 2007; 46: 7548-7558.

3 Carino IS, Pasqua L, Testa F, Aiello R, Puoci F, lemma F et al. Silica-based mesoporous materials as drug delivery system for methotrexate release. Drug Deliv 2007; 14: 491-495.

4 Maynard AD. Nanotechnology: the next big thing, or much ado about nothing? Ann Occup Hyg 2007; 51: 1-12.

5 Nemmar A, Al-Salam S, Zia S, Yasin J, Al Husseni I, Ali BH. Diesel exhaust particles in the lung aggravate experimental acute renal failure. Toxicol Sci 2010; 113: 267-277.

6 Inoue K, Koike E, Takano H. Comprehensive analysis of elastase-induced pulmonary emphysema in mice: effects of ambient existing particulate matters. Int Immunopharmacol 2010; 10: 1380-1389.

7 Inoue K, Takano H, Yanagisawa R, Ichinose T, Shimada A, Yoshikawa T. Pulmonary exposure to diesel exhaust particles induces airway inflammation and cytokine expression in NC/Nga mice. Arch Toxicol 2005; 79: 595-599.

8 Nishimori H, Kondoh M, Isoda K, Tsunoda S, Tsutsumi Y, Yagi K. Silica nanoparticles as hepatotoxicants. Eur J Pharm Biopharm 2009; 72: 496-501.

9 Nishimori $\mathrm{H}$, Kondoh M, Isoda K, Tsunoda S, Tsutsumi Y, Yagi K. Histological analysis of $70-\mathrm{nm}$ silica particles-induced chronic toxicity in mice. Eur J Pharm Biopharm 2009; 72: 626-629.

10 Chen W, Zhang Q, Kaplan BL, Baker GL, Kaminski NE. Induced T cell cytokine production is enhanced by engineered nanoparticles. Nanotoxicology 2014; 8(Suppl 1): 11-23.

11 Kuhlbusch TA, Asbach C, Fissan H, Gohler D, Stintz M. Nanoparticle exposure at nanotechnology workplaces: a review. Part Fibre Toxicol 2011 8: 22.

12 Eisen EA, Costello S, Chevrier J, Picciotto S. Epidemiologic challenges for studies of occupational exposure to engineered nanoparticles; a commentary. J Occup Environ Med 2011; 53: S57-S61.

13 Inoue $\mathrm{K}$, Takano $\mathrm{H}$. Aggravating impact of nanoparticles on immunemediated pulmonary inflammation. ScientificWorldJournal 2011; 11: 382-390.

14 Sanya RE, Kirenga BJ, Worodria W, Okot-Nwang M. Risk factors for asthma exacerbation in patients presenting to an emergency unit of a national referral hospital in Kampala, Uganda. Afr Health Sci 2014; 14: 707-715.

15 Saraya T, Kurai D, Ishii H, Ito A, Sasaki Y, Niwa S et al. Epidemiology of virus-induced asthma exacerbations: with special reference to the role of human rhinovirus. Front Microbiol 2014; 5: 226.
16 Esposito S, Tenconi R, Lelii M, Preti V, Nazzari E, Consolo S et al. Possible molecular mechanisms linking air pollution and asthma in children. $B M C$ Pulm Med 2014; 14: 31.

17 Dombu C, Carpentier R, Betbeder D. Influence of surface charge and inner composition of nanoparticles on intracellular delivery of proteins in airway epithelial cells. Biomaterials 2012; 33: 9117-9126.

18 Chau E, Galloway JF, Nelson A, Breysse PN, Wirtz D, Searson PC et al. Effect of modifying quantum dot surface charge on airway epithelial cell uptake in vitro. Nanotoxicology 2013; 7: 1143-1151.

19 Marzaioli V, Aguilar-Pimentel JA, Weichenmeier I, Luxenhofer G, Wiemann $M$, Landsiedel $\mathrm{R}$ et al. Surface modifications of silica nanoparticles are crucial for their inert versus proinflammatory and immunomodulatory properties. Int J Nanomedicine 2014; 9: 2815-2832.

20 Cullinan P, Reid P. Pneumoconiosis. Prim Care Respir J 2013; 22: 249-252.

21 Laney AS, Weissman DN. The classic pneumoconioses: new epidemiological and laboratory observations. Clin Chest Med 2012; 33: 745-758.

22 Steenland K, Ward E. Silica: a lung carcinogen. CA Cancer J Clin 2014; 64: 63-69

23 Caplan A. Rheumatoid disease and pneumoconiosis (Caplan's syndrome) Proc R Soc Med 1959; 52: 1111-1113.

24 Yamazaki S, Yoshiike F, Hirai K, Kakegawa T, Ikeda M, Nagata A et al. Silica-associated systemic lupus erythematosus in an elderly man. Intern Med 2007; 46: 1867-1871.

25 Suh WH, Suslick KS, Stucky GD, Suh YH. Nanotechnology, nanotoxicology, and neuroscience. Prog Neurobiol 2009; 87: 133-170.

26 Li FF, Zhang XX, Guo SY, Gan Y, Li J. [Preliminary study on pH-sensitive lipid bilayer-coated mesoporous silica nanoparticles as a novel drug carrier for antitumor drug]. Yao Xue Xue Bao 2013; 48: 291-297.

27 Luo GF, Chen WH, Liu Y, Lei Q, Zhuo RX, Zhang XZ. Multifunctional enveloped mesoporous silica nanoparticles for subcellular co-delivery of drug and therapeutic peptide. Sci Rep 2014; 4: 6064.

28 Andreani T, de Souza AL, Kiill CP, Lorenzon EN, Fangueiro JF, Calpena AC et al. Preparation and characterization of PEG-coated silica nanoparticles for oral insulin delivery. Int J Pharm 2014; 473: 627-635.

29 Han B, Guo J, Abrahaley T, Qin L, Wang L, Zheng Y et al. Adverse effect of nano-silicon dioxide on lung function of rats with or without ovalbumin immunization. PLoS One 2011; 6: e17236.

30 Brandenberger C, Rowley NL, Jackson-Humbles DN, Zhang Q, Bramble LA, Lewandowski RP et al. Engineered silica nanoparticles act as adjuvants to enhance allergic airway disease in mice. Part Fibre Toxicol 2013; 10: 26.

31 Neunkirchner A, Leb-ReichI VM, Schmetterer KG, Mutschlechner S, Kueng HJ, Haiderer D et al. Human TCR transgenic Bet v 1-specific Th1 cells suppress the effector function of Bet $v$ 1-specific Th2 cells. J Immunol 2011; 187: 4077-4087.

$32 \mathrm{He} \mathrm{Q}$, Zhang Z, Gao F, Li Y, Shi J. In vivo biodistribution and urinary excretion of mesoporous silica nanoparticles: effects of particle size and PEGylation. Small 2011; 7: 271-280.

33 Docter D, Bantz C, Westmeier D, Galla HJ, Wang Q, Kirkpatrick JC et al. The protein corona protects against size- and dose-dependent toxicity of amorphous silica nanoparticles. Beilstein J Nanotechnol 2014; 5: 1380-1392.

34 Brown DM, Kanase N, Gaiser B, Johnston H, Stone V. Inflammation and gene expression in the rat lung after instillation of silica nanoparticles: effect of size, dispersion medium and particle surface charge. Toxicol Lett 2013.

35 Skuland T, Ovrevik J, Lag M, Refsnes M. Role of size and surface area for pro-inflammatory responses to silica nanoparticles in epithelial lung cells: importance of exposure conditions. Toxicol In Vitro 2014; 28: 146-155.

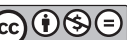

This work is licensed under a Creative Commons Attribution-NonCommercial-NoDerivs 4.0 International License. The images or other third party material in this article are included in the article's Creative Commons license, unless indicated otherwise in the credit line; if the material is not included under the Creative Commons license, users will need to obtain permission from the license holder to reproduce the material. To view a copy of this license, visit http://creativecommons.org/licenses/by-nc-nd/4.0/ 